\author{
MITSUBISHI ELECTRIC RESEARCH LABORATORIES \\ http://www.merl.com
}

\title{
Integrated Design and Control of Flexure-Based Nanopositioning Systems - Part I: Methodology
}

\author{
Shilpiekandula, V.; Youcef-Toumi, K.
}

TR2011-053 August 2011

\begin{abstract}
Flexure-based mechanisms, also referred to as flexures, are widely being used as motion-guidance, or bearing, elements in applications requiring multi-degree-of-freedom positioning and alignment. Unlike friction-bearings (such as sliding or rolling contact bearings), flexures can be designed to offer, to a large extent, reliable linear elastic motion with a high resolution (on the order of nanometers) over small ranges of motion (on order of micrometers). Example applications include positioning a probe or sample in atomic force microscopy, alignment of tool and sample in stamping processes, and fine-positioning of wafer steppers in semiconductor manufacturing. These applications are often required satisfy critical functional requirements, such as load-capacity, bandwidth, resolution, and range. A systematic approach is needed to simultaneously address the design and control challenges involved, starting from the initial design concept generation stage to the final control implementation and testing. In this paper, we present an integrated design and control method for implementing flexurebased nanopositioning systems. We discuss the need for varying design topology and order of a controller in design and control optimization. An automation engine generates a set of flexurebased design topologies and also controllers of varying order in the optimization. A simple 1-DOF example is worked out to illustrate the steps involved in using this methodology. The outcome of the exercise is a novel design topology, with it shape and size optimized, and a controller synthesized such that a desired control bandwidth and design requirements of strength and modal separation are met.
\end{abstract}

World Congress of the International Federation of Automatic Control

This work may not be copied or reproduced in whole or in part for any commercial purpose. Permission to copy in whole or in part without payment of fee is granted for nonprofit educational and research purposes provided that all such whole or partial copies include the following: a notice that such copying is by permission of Mitsubishi Electric Research Laboratories, Inc.; an acknowledgment of the authors and individual contributions to the work; and all applicable portions of the copyright notice. Copying, reproduction, or republishing for any other purpose shall require a license with payment of fee to Mitsubishi Electric Research Laboratories, Inc. All rights reserved.

Copyright (C) Mitsubishi Electric Research Laboratories, Inc., 2011

201 Broadway, Cambridge, Massachusetts 02139 



\title{
Integrated Design and Control of Flexure-Based Nanopositioning Systems - Part I: Methodology
}

\author{
Vijay Shilpiekandula ${ }^{1}$ and Kamal Youcef-Toumi \\ Mechatronics Research Laboratory, \\ Department of Mechanical Engineering, \\ 77 Massachusetts Avenue, Cambridge MA 02139
}

\begin{abstract}
Flexure-based mechanisms, also referred to as flexures, are widely being used as motion-guidance, or bearing, elements in applications requiring multi-degree-of-freedom positioning and alignment. Unlike friction-bearings (such as sliding or rolling contact bearings), flexures can be designed to offer, to a large extent, reliable linear elastic motion with a high resolution (on the order of nanometers) over small ranges of motion (on order of micrometers). Example applications include positioning a probe or sample in atomic force microscopy, alignment of tool and sample in stamping processes, and fine-positioning of wafer steppers in semiconductor manufacturing. These applications are often required satisfy critical functional requirements, such as load-capacity, bandwidth, resolution, and range. A systematic approach is needed to simultaneously address the design and control challenges involved, starting from the initial design concept generation stage to the final control implementation and testing. In this paper, we present an integrated design and control method for implementing flexurebased nanopositioning systems. We discuss the need for varying design topology and order of a controller in design and control optimization. An automation engine generates a set of flexurebased design topologies and also controllers of varying order in the optimization. A simple 1-DOF example is worked out to illustrate the steps involved in using this methodology. The outcome of the exercise is a novel design topology, with it shape and size optimized, and a controller synthesized such that a desired control bandwidth and design requirements of strength and modal separation are met.
\end{abstract}

Keywords: Flexure-based mechanisms, Nanopositioning, Topology Generation, Synthesis.

\section{INTRODUCTION}

Precision linear positioning and angular (rotary) alignment at nanoscale resolutions are often referred to as "nanopositioning." Many applications for nanopositioning systems have emerged over the past few decades in various contexts, such as semiconductor manufacturing, metrology, x-ray crystallography, and biological imaging. From among the many different methods of implementing nanopositioning systems, those involving compliant flexure-based mechanisms have gained popularity over the years. Flexure-based mechanisms are composed of slender beam-like spring elements in their mechanical design; they are close to being ideal motion bearings with minimal friction, backlash, and other uncertainties. These advantages make flexure-based mechanisms, also referred to as flexures, ideal candidates for precision motion control implementations.

\footnotetext{
1 Corresponding author: svijay@mit.edu
}

The drive for better performance steers high-resolution designs towards satisfying stringent specifications in terms of functional parameters such as range, load-capacity, and bandwidth. While flexure-based nanopositioning systems for such advanced nanotechnology applications have been around for the past few decades [8], designing them for dynamic performance has received little attention. Kinematic arrangement of parallel flexure systems using projection geometry theory has been worked out in [9]. Analysis of the statics [7] and dynamics of flexure-based mechanisms have been extensively studied $[11,33]$. However, few publications $[12,13]$ have appeared in the context of design for dynamic performance. The design of flexures in the context of mechanical advantage is detailed in [12]. A finite-element approach based on Euler-Bernoulli beam bending theory is formulated for analyzing dynamics in [13] and optimizing the design space for precision flexure-based applications in [14].

While dynamic performance of just the flexure-based mechanism or 'plant' presents one of the performance requirements, a more challenging and critical requirement 
is achieving an overall desired closed-loop control performance [15] of a system assembled with the mechanism, and suitable actuator and sensor subsystems. A potentially useful approach in this context should be based on integrating design and control methods right from design conception and validation phase before hardware implementations are tested out. To the best of our knowledge, an integrated approach for the design and control of flexurebased nanopositioning systems is lacking in the existing literature. A common systems-based methodology can facilitate developing valuable synthesis tools for achieving the desired closed-loop control performance.

In this paper, we tackle the "co-design" problem, integrating design and control for achieving a desired closedloop control performance of flexure-based nanopositioning systems. In Section 2 we provide a detailed review of relevant literature that tackle the co-design problem, while (i) motivating the need for co-design from two practical servohardware examples, and (ii) highlighting the deficiencies in current approaches in the field of nanopositioning systems. A novel method for integrated design and control is presented in Section 3, and tailored for flexure-based mechanisms. A detailed set of steps needed to implement the method is analyzed in Section 4. The paper concludes with a summary in Section 5. The reader is referred to Part II of this paper for an application case study of a 1-DOF alignment mechanism that is worked out in detail using the proposed method.

\section{INTEGRATED DESIGN AND CONTROL}

Integrated design and control has been an active area of research spanning applications such as robotic manipulator design and control [17]-[22], motion stages developed using lead-screw drives [24], passive and active vibration isolation platforms $[25,26]$, and chemical process control [27]. In this section, we cover a detailed survey of relevant methods in the literature.

\subsection{Varying Design and/or Control Parameters}

In what follows, we first review works reported on optimizing a design (plant) or controller so that a desired performance metric (design or control) is met under physical (design) constraints, and state/output and control constraints.

Optimal design and control of flexible structures has been studied for (i) improving a mass efficiency metric (defined as mass moved per unit work output) in [3], (ii) a quadratic control performance index in [6], and (iii) a weighted sum of structural mass and the energy of the controlled mechanism in [26]. The integrated design and control problem was formulated as a multi-objective optimization involving design and proportional-integral-derivative (PID) controller parameters in [28] for mechatronic systems. A similar approach optimizing proportional-derivative (PD) controller parameters and design parameters for four-bar linkages was studied in [18]. A non-linear optimization formulation including design costs and a robust performance constraint on the weighted sum of sensitivity and complementary sensitivity functions is considered for a chemical distillation column in [27]. Decentralized control techniques were used to solve for the optimization of passive (design parameters) and active (control parameters) for vibration isolation platforms in [25]. Different approaches for integrated design and control have been studied from an optimization theory standpoint in [17] and [15]. These approaches include (i)sequential optimization with design optimization followed by control optimization, (ii)simultaneous design and control optimization, and (iii)an iterative combination where the design is initially optimized without affecting the controller, then the controller is optimized, and such a cycle is iterated until performance requirements are met.

Optimal locations for embedded actuators and sensors in a mechanism with distributed compliance are discussed in [16] for satisfying controllability and observability conditions. However, neither the design of the controller nor the influence of a poor design choice on control performance is addressed in this reference. A related critical issue is one of lightly damped flexible modes of flexure-based mechanisms. Physical damping is low in flexures made from metals such as aluminium (used in development stages of the design process for ease of machining), or titanium (used in the implementation and testing phase because of its high fatigue strength and other material properties). External damping such as squeeze film damping and foamdamping have been suggested and explored for flexures in the past. Active damping through appropriate selection of control strategies needs to be addressed to tackle the lightly damped resonances in these structures. Since the level of damping in an assembled mechanism is hard to predict before the fabricated product is available for testing, it becomes necessary to iterate the design process with thorough system identification and testing of hardware mechanism implementations.

Motion stages developed using lead-screw drives were characterized for their dynamics and controlled with classical lead-lag compensators in [24]. In this reference, the design and control performance space in terms of performance requirements, such as (i) the positioning error and (ii) control bandwidth of the drive and (iii) the maximum acceleration of the carriage, were captured for the entire range of geometry, material, and other parameters. Since lightly damped harmonics hinder control performance, achieving robust passive damping with foam-based materials is proposed by the same research group in [38]. An integrated design and control methodology for high-speed control of robotic manipulators is presented in $[21,22]$. Since unmodeled dynamics in the control bandwidth can adversely affect the performance, it is necessary to account for model-truncation errors in the design and control optimization. In this context, a constraint condition on the Hankel norm of the truncated modes is formulated in the optimization problem [21]. 


\subsection{Varying Design Topology}

Unlike most of the methods reported above, few references address changing the design structure or configuration (referred to as the topology) itself, so that control performance is enhanced. We examine here two specific cases from the literature that illustrate the importance of selecting an appropriate design topology before deploying any optimization routine.

Consider the example of a robotic system shown in Fig. 1 addressing the end-point control of a flexible link. The actuator is a rotary servomotor that generates a torque required for moving the end-point of the link. The feedback signal is the end-point position, which can be recorded by a sensor such as an accelerometer. Since the actuator and the sensor are not at the same location in space, i.e. the system is non-collocated. For the non-collocated system, the flexibility of the link is known to cause nonminimum phase zeros in the transfer function between the voltage applied to the motor and the measured end-point displacement [36].

In order to avoid the occurrence of the non-minimum phase zero, the actuation point shown in Fig. 1 (a) can be moved away from the motor closer to the end-point, as shown in Fig. 1(b). With the actuation location moved closer to the end-point, the portion of the link from the new actuation point to the sensor location is shorter, and hence stiffer. It is shown in [36] that, under certain geometry conditions, this topology change results in moving the zeros from the real-axis on to the imaginary axis, making the system minimum-phase. The design topology change shown in Fig. 1(b) is implemented in Fig. 1(c) using a cable transmission from the motor.

Without this topology change, with the actuator just as the motor and sensor at the end-point, the system would be non-minimum phase and pose critical control challenges. $^{2}$

We next consider the example of a hard disk drive actuator subsystem in Fig. 2. As shown in Fig. 2 (a), this subsystem positions the read (or write) head at the end of an arm pivoting about a rotary bearing. A lorentz-force $F_{m}$ generated by voice coil motor at an distance $R_{e}$ causes the arm to rotate about the pivot. However, the applied force $F_{m}$ also exerts a force $F_{r}$ at the bearing, exciting its translation mode. The displacement at the read head is composed of the difference of modal responses arising from the rigid body rotation and the bearing translation mode.

The presence of the bearing translation mode is undesirable for two reasons: (i) the translation shows up in the displacement at the read head and (ii) the transfer function between the applied force and the measured displacement at read head can be non-minimum phase under certain

2 The constraint on control bandwidth imposed by non-minimum phase zeros is worked out for an example positioning system in Part II of this paper.

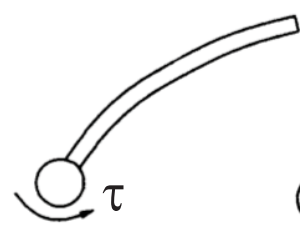

(a)

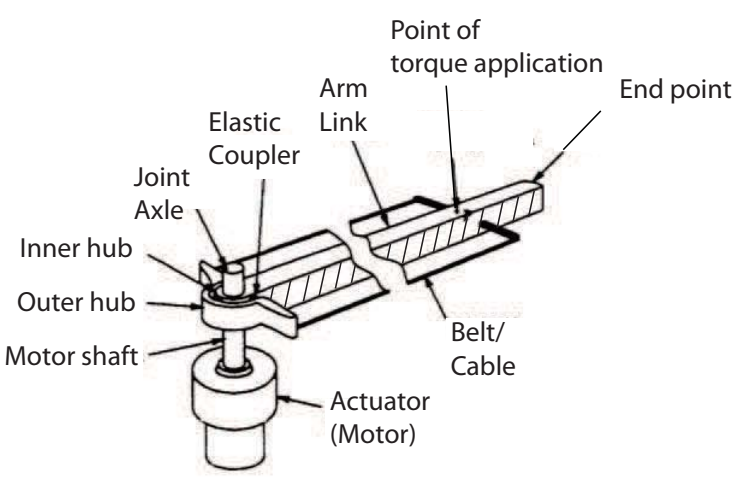

(c)

Fig. 1. Design for control example from [36]. (a) Moving the torque application point away from the hub and closer to the end point of the flexible manipulator results in minimum-phase dynamics, and hence allows for higher control bandwidths. (b) A belt transmission is used on a motor to vary the location of the torque application point.

geometry conditions [37]. A novel actuator (see Fig. 2(b)) based on a set of magnetic arrays called Hallbach arrays is designed in [35] to form a voice coil motor that generates only a torque and now net translational force. The new design topology is shown in Fig. 2(c) with the purelytorque motor mounted in the pivot itself, without the need for the linear force $F_{m}$ applied at the arm distance $R_{e}$.

Without this design topology change, the translation of the bearing and the non-minimum phase zero would limit the performance of the read head.

In summary, the two examples discussed above emphasize the need for developing suitable design topologies before any optimization is attempted. An interesting extension of this problem is one of identifying a set or library of topologies from which we can select an appropriate topology.

In what follows, we discuss our integrated design and control method that is based on optimizing over a library of topologies, not just dimensional (and other) parameters within a given topology. 


\section{CONFIDENTIAL. Limited circulation. For review only.}
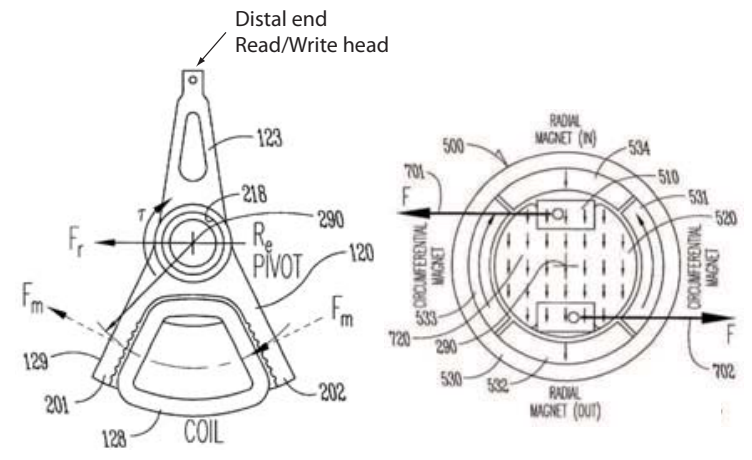

(a)

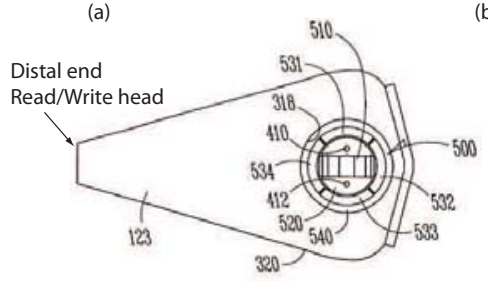

(c)

Fig. 2. Design for control example from [35] modifying actuator design to eliminate translational loading in voice-coil motors. (a) In disk drives, the actuator for the read/write head is a typical lorentz-force voice coil motor that produces a force at an offset. The force also excites the translational mode of the bearing. (b) A novel design for the voice coil motor, based on a magnetic array called as Hallbach array, which is commonly found in linear motors, is used in a rotary configuration to produce a unidirectional magnetic field in the hub. (c) The resulting actuator is a pure torque motor that minimizes the effect of the translational mode of the bearing.

\section{PROPOSED METHOD}

Based on the examples of integrated design and control described in Section 2, we identify the four possible cases for integrated design and control in Table 1. As indicated in the table, in any design for control approach, the design (or plant) and the controller need to be judiciously chosen in the problem formulation step. The options listed in the table are based either on a fixed or a varying topology/order for a design/controller. Before we proceed any further, we present our definitions of these terms as relevant to the integrated design and control methodology we will propose shortly.

A fixed controller is one with a pre-specified order and parameters to be selected appropriately. A basic knowledge of the plant dynamics can facilitate a nominal choice for the controller order. The problem of maximizing performance reduces to selecting the best possible controller parameters. However, such a fixed structure for the controller limits in most cases the freedom in maximizing performance. On the other hand, we define a varying order controller as one in which the order is not pre-selected. Rather, the controller order evolves in the integrated design and control iterations.

Similarly, we define a fixed design topology as one in which the overall structure is initially chosen and the iterations are performed to tune the parameters. Tuning the parameters of a fixed design cannot alter the design structure at all. On the other hand, by a varying design topology option, we iterate with changes in design topology, or configuration, each time creating a different design altogether. For example, a design topology can be varied to go from a parallel kinematic design to a serial kinematic design, or from an exact-constraint design to an elastic-averaging design.

Table 1. All possible cases for integrated design and control.

\begin{tabular}{|l|l|l|}
\hline Case & Design Topology & Controller Order \\
\hline I & Fixed & Fixed \\
II & Fixed & Allowed to Vary \\
III & Allowed to Vary & Fixed \\
IV & Allowed to Vary & Allowed to Vary \\
\hline
\end{tabular}

The cases presented in the table work out as follows. In Case I, the integrated approach optimizes performance over a combination of design and controller parameters. The final outcome after the iterations is a design and a controller of the same structure as at the beginning of the iterations, but with the selection of the most promising parameters. Hence, in this case, both design and control structure are fixed and cannot be altered. A poor choice of design topology or controller structure can leave critical performance requirements unfulfilled.

In Case II, for a fixed design topology, the controller is allowed to vary. Hence, in the iterations, the design parameters and controller order evolve to facilitate optimizing the performance.

In Case III, the design topology is varied (i.e. many possible design structures are tested) against a controller with a fixed structure. This case fundamentally limits the performance, since for every new plant we are confined to the same controller type. The potential performance of the system can be lost in thus fixing the control structure.

The most intuitive and useful case is Case IV, which uses a varying the design topology and the order of the controller. However, since the number of possible design configurations in typical nanopositioning system applications are finite, the varying design topology problem can be broken down into a number of fixed design (each tested with a controller of varying order) problems. Hence, we formulate our methodology on Case II with a controller of varying order tested for each fixed design topology of all possible design topologies.

\section{DETAILED STEPS}

In this section, we detail the steps involved in implementing the integrated design and control approach based on Case IV of Table 1, in which both the design topology and 
the controller structure are allowed to vary. As discussed above, we simplify this to the one of Case II for a varying order controller tested for all possible fixed design topologies from a design library. The methodology is illustrated as a flow chart diagram in Figure 4.

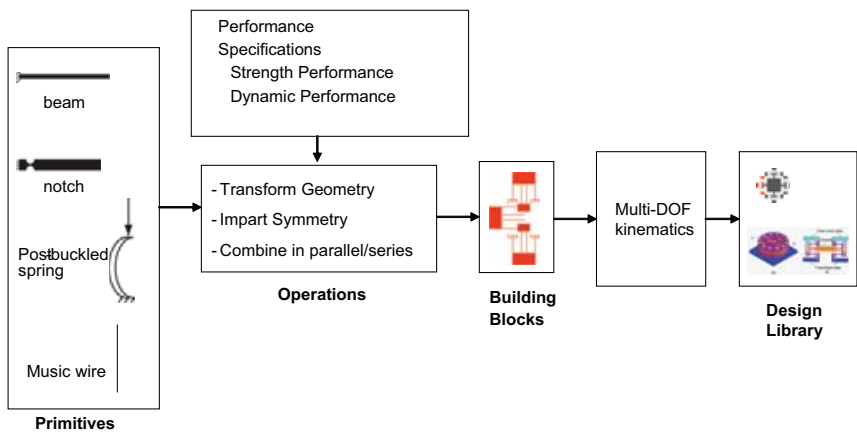

Fig. 3. A performance-driven design library shown as constructed from building blocks prepared by performance-driven operations on a set of primitives. Novel designs synthesized with this method are schematically shown in the Design Library block.

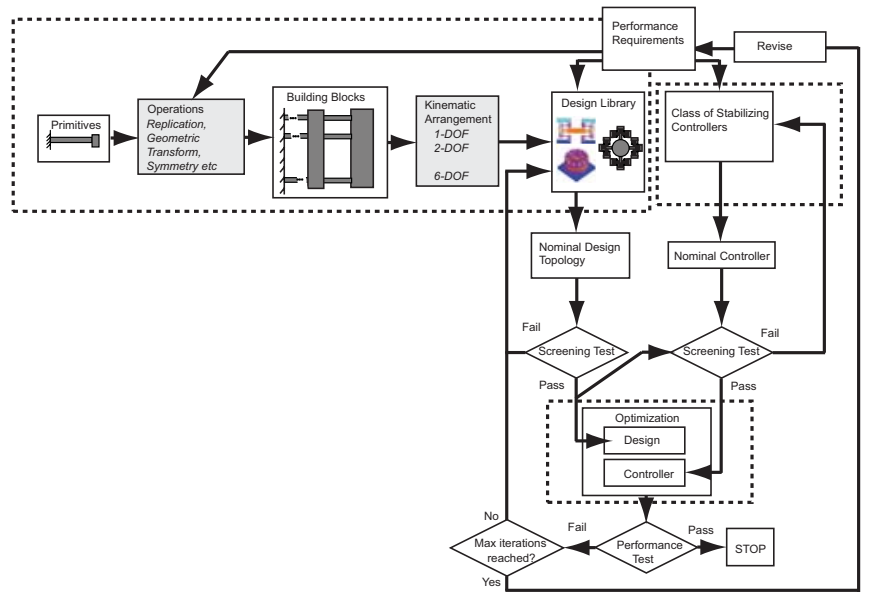

Fig. 4. Integrated design and control methodology for meeting performance requirements.

screening test is necessary before (blindly) feeding the design topology to a shape and size optimization procedure.

Step 1: Performance Specification: Formulate performanceStep 4: Controller Selection/Screening: On the controller requirements for the flexure-based nanopositioning system. These requirements can be for the structural, thermal, or control, and other aspects of performance.

Step 2: Design Topology Library Generation: The following actions are involved in automating generation of topology concepts that improve the specified performance requirements. (i)First, a library is set up with a set of primitives.

(ii) These primitives are subjected to a finite number of operations dictated by performance requirements. These operations could be, for example, a parallel orStep 5: serial replication, or a geometrical transformation, or adding a redundant constraint that imparts symmetry.

(iii) The primitives are then subjected to these operations generate building blocks that meet the desired performance requirement.

(iv) Once the building blocks are generated, a library of design topologies can be generated by using the building block as an implementation of the constraints (following a constraint-based synthesis approach [29]) for satisfying the necessary kinematics.

In a nutshell, using the performance-tuned building block allows to meet a strength, or modal performance criterion, while the constraint-based arrangement allows for satisfying the required kinematics. This step is detailed for the example 1-DOF positioning system in Part II of this paper.

Step 3: Design Topology Selection/Screening: Every nominal design topology in the topology library is subject to a screening test to eliminate design topologies that obviously do not meet critical requirements. This side, an initial controller is selected as a nominal controller from the entire class of stabilizing controllers for the screened nominal design topology. For the screened nominal design and the nominal controller selected above, a screening test is used to weed out controller choices that do not allow for critical requirements to be met. It is important to perform this screening test before (blindly) feeding the design to an optimization procedure. The structure of the nominal controller is revised until it passes the screening test.

5: Optimization: Given that the nominal design and the nominal controller have passed the screening test, we now feed them to an optimization procedure. This procedure collects the design and controller parameters and optimizes them for an objective function defined by the user. The design optimization may target shape and size optimization of the chosen topology. The controller optimization varies the order of the controller to meet the robust stability and performance specifications on sensitivity transfer function or complementary sensitivity transfer function.

Many choices exist for implementing the design and control optimization. As we discussed in Section 2, different approaches for the optimization are discussed in [15]. These include (i) simultaneous design and control optimization (ii) sequential optimization, with design optimization followed by control optimization, and (iii) iterative design and control optimization. While each of these approaches could be applied in our methodology, we select the option (ii) of optimizing design first and then optimizing the controller for the optimized design since (i) it comes closest to what is done in practice when designing 
and controlling a hardware positioning system and (ii) it is computationally less intensive and feasible. The design optimization is formulated to select the design parameters that best allow minimizing or maximizing a desired cost function while meeting design constraints such as stress, fatigue limits. This part of the design optimization is often referred to as shape and size optimization. The optimized plant is fed to the controller optimization block. Once a nominal controller is chosen, it can be enhanced for imparting robustness.

If the performance requirements are met by the outcome of the optimization procedure, the controller is tested on the hardware to see if the performance can be demonstrated. If the performance requirements are not met at the end of the optimization procedure and the maximum number of iterations has not been reached, the nominal design topology is revised. Unless the nominal design topology is revised it is impossible to achieve the desired performance. If the maximum number of iterations has been reached, the only way to proceed any further is by relaxing the performance requirements. The design intuition gained from the optimization or from the hardware application should be used to revise the performance requirements suitably, taking us back to Step 1 listed above.

\section{SUMMARY}

In this paper, we presented a method for iterating on design (plant) topologies and controller order to achieve a desired closed-loop system specification. It is emphasized that iterating a design is not just about fine-tuning shape and size of a particular design configuration. Instead, we need to iterate over design topologies and controller order. An automated topology generation engine is discussed. Further, a novel controller parameterization is used to vary the controller order while directly tuning the sensitivity function to a desired form. An example of a flexure-based $1-\mathrm{DOF}$ positioning system is worked out in Part II of this paper as an application of the integrated design and control methodology.

\section{Acknowledgements:}

The first author is thankful to Xerox Foundation and MIT Dean School of Engineering for fellowship support. We are thankful to Dr. Pradeep Subrahmanyan of Data Physics Corporation for discussion of design and control interactions in precision machines.

\section{REFERENCES}

[1] White JR, "The nanogate: nanoscale flow control," Ph.D. dissertation, Cambridge, MA: Massachusetts Institute of Technology, Department of Mechanical Engineering, June 2003.

[2] Shilpiekandula V, "Progress through Mechanics: Small-scale Gaps," Mechanics (Publication of the American Academy of Mechanics), vol. 35, no. 9-10, pp. 3-6, Sep-Oct 2006.
[3] Prechtl EF and Hall SR, "Design of a High Efficiency, Large Stroke, Electro-Mechanical Actuator," Journal of Smart Materials and Structures, vol. 8, pp. 13-30, 1999.

[4] Shilpiekandula V, Youcef-Toumi K, "Modeling and Control of a Programmable Filter for Separation of Biologically Active Molecules," In Proceedings of American Control Conference, pp. 394-399, June 2005.

[5] Slocum AH, "Precision Machine Design," Englewood Cliffs, NJ, Prentice Hall, 1992.

[6] Canfield RA and Meirovitch L, "Integrated structural design and vibration suppression using independent modal-space control," AIAA/ASME/ASCE/AHS/ASC Structures, Structural Dynamics, and Materials Conference, 34th and AIAA/ASME Adaptive Structures Forum, La Jolla, CA, Technical Papers. Pt. 6 (A93-33876 13-39), pp. 3404-3414, April 1993.

[7] Awtar S, Slocum AH, Sevincer E. "Characteristics of Beam-based Flexure Modules," Journal of Mechanical Design, vol. 129 (6): 625-639 Jun 2007.

[8] Jones RV, "Instruments and Experiences: Papers on Measurement and Instrument Design," John Wiley, 1988.

[9] Hopkins JB, "Design of Parallel Flexure Systems via Freedom and Constraint Topologies," Master of Science Thesis, Massachusetts Institute of Technology, 2005.

[10] White J, Ma H, Lang J, Slocum AH, "An Instrument to Control Parallel Plate Separation for Nanoscale Flow Control," Review of Scientific Instruments, vol. 74, no. 11, November 2003.

[11] Lobontiu N, "Compliant Mechanisms : Design of Flexure Hinges," Boca Raton, CRC Press, 2003

[12] Li Z, and S. Kota, "Dynamic Analysis of Compliant Mechanisms, "Proceedings of DETC, 27th Biannual Mechanisms and Robotics Conference," Sep 29 - Oct 2, Montreal, Canada, 2002.

[13] Choi KB, "Dynamics of a Compliant Mechanism based on Flexure Hinges," Proceedings of the Institution of Mechanical Engineers, Part-C, Journal of Mechanical Engineering Science 219 (2): 225-235 Feb 2005.

[14] Choi KB, Han CS, "Optimal Design of a Compliant Mechanism with Circular Notch Flexure Hinges," Proceedings of the institution of Mechanical Engineers, Part-C, Journal of Mechanical Engineering Science 221 (3): 385-392 Mar 2007.

[15] Fathy HK, Reyer JA, Papalambros PY, Ulsoy AG, "On the Coupling between the Plant and Controller Optimization Problems," In Proceedings of American Control Conference, Arlington VA, June 25-27, 2001.

[16] Trease BP, Kota S, "Synthesis of Adaptive and Controllable Compliant Systems with Embedded Actuators and Sensors," In Proceedings of DETC 2006, International Design Engineering Technical Conference, Philadelphia, PA, Sept 10-13, 2006. 
[17] Fu K, Sun D, Mills JK, "Simultaenous Mechanical Structure and Control System Design: Optimization and Convex Approaches," In Proceedings of IEEE International Symposium on Intelligetn Control, Vancouver, Canada, October 27-30, 2002.

[18] Wu FX, Zhang WJ, Li Q, Quyang PR, "Integrated Design and PD Control of High-Speed Closed-loop Mechanisms," Journal of Dynamic Systems Measurement and Control, vol. 124, (4), pp. 522-528, Dec 2002.

[19] Pil A and Asada HH, "Rapid Recursive Structure ReDesign for Improved Dynamic Performance", ASME Journal of Dynamic Systems, Measurement and Control, July 1994, Vol. 117, December 1995.

[20] Rai S, Asada HH, "Computer-Aided Structure Modification of Electromechanical Systems Using Singular Value Decomposition", ASME J. Mechanical Design, Vol. 116, December 1994.

[21] Savant SV, Asada HH, "Integrated Structure/Control Design Based on Model Validity and Robustness Margin," In Proceedings of American Control Conference, vol. 4, pp. 2871-2875, June 2-4, 1999.

[22] Savant SV, Asada HH, "Shaping Structure Dynamics with Truncation-Error Bounded Reduced-Order Models for Integrated Mechanism/Control Design," In Proceedings of American Control Conference, vol. 6, pp. 3540-3544, June 24-26, 1998.

[23] Savant SV, "Structure Design Fpor Control Robustness Against Truncation Uncertainty," Ph.D. Thesis, Department of Mechanical Engineering, MIT, 1999.

[24] Varanasi KK, "On the Design of a Precision Machine for Closed-loop Performance," M.S. Thesis, Department of Mechanical Engineering, Massachusetts Institute of Technology, Cambridge MA 02139, USA.

[25] Zuo L, "Element and System Design for Active and Passive Vibration Isolation," Ph.D. Thesis, Department of Mechanical Engineering, Massachusetts Institute of Technology, Cambridge MA 02139, USA.

[26] Xianmin Z, Jianwei L, Yunwen S, "Simultaneous Optimal Structure and Control Design of Flexible Linkage Mechanism for Noise Attentuation," Journal of Sound and Vibration, vol. 299, pp. 1124-1133, 2007.

[27] Chawankul N, Budman H, Douglas PL, "The Integration Of Design and Control: Internal Model Control And Robustness," Computers and Chemical Engineering vol. 29 (2), pp. 261-271, Jan 15, 2005.

[28] da Silva MM, Bruls O, Desmet W, Van Brussel H, "Integrated structure and control design for mechatronic systems with configuration-dependent dynamics," Mechatronics, vol. 19, (6), pp. 1016-1025, Sep 2009.

[29] Awtar S, "Synthesis and Analysis of Parallel Kinematic XY Flexure Mechanisms," Ph.D. Thesis, Massachusetts Institute of Technology, Cambridge MA 02139.

[30] Awtar S and Sevincer E, 2006, "Elastic Averaging in Flexure Mechanisms: A Multi-Parallelogram Flexure Case-study," In Proceedigns of ASME IDETC/CIE 2006, Philadelphia, PA, Paper No. 99752.
[31] Trease BP, Moon Y, Kota S, "Design of Large Displacement Compliant Joints," Journal of Mechanical Design, vol. 127, pp 788-798, July 2005.

[32] Smith ST, "Flexures: elements of elastic mechanisms," Amsterdam, Gordon and Breach, 2000.

[33] Howell LL, "Compliant mechanisms," New York , Wiley 2001.

[34] Pil A and Asada HH, "Integrated Structure/Control Design of Mechatronic Systems", IEEE/ASME Transactions on Mechatronics, September 1996.

[35] Subrahmanyan PK, "Moving Magnet Voice Coil Motor Using Halbach Arrays," US Patent 703116B2.

[36] Park JH, Asada H, "Design and control of minimumphase flexible arms with torque transmission mechanisms," IEEE International Conference on Robotics and Automation, pp 1790-1795, May 1990.

[37] Subrahmanyan PK, "Control and design interactions in precision machines," Tutorial Notes, American Society for Precision Engineering Annual Conference, Portland OR, Oct 2009.

[38] Varanasi KK and Nayfeh SA, "Damping of Flexural Vibration Using Low-Density, Low-Wave-Speed Media," Journal of Sound and Vibration, vol. 292, pp. 402-414, 2006.

[39] Varanasi KK, Nayfeh SA, "The Dynamics of LeadScrew Drives: Low-Order Modeling and Experiments," Journal of Dynamic Systems, Measurement, and Control, Transactions of the ASME, vol. 126, pp. 388-396.

[40] Skogestad S and Postlethwaite I, "Multivariable feedback control : analysis and design," Hoboken, NJ, John Wiley, 2005. 


\title{
Integrated Design and Control of Flexure-Based Nanopositioning Systems - Part II: Application Case Study
}

\author{
Vijay Shilpiekandula ${ }^{1}$ and Kamal Youcef-Toumi \\ Mechatronics Research Laboratory, \\ Department of Mechanical Engineering, \\ 77 Massachusetts Avenue, Cambridge MA 02139
}

\begin{abstract}
Flexure-based mechanisms contain slender beam-like modules that undergo linear elastic deflections over small ranges of motion at nanoscale resolutions. They are hence often used as bearing elements in nanopositioning systems, along with precision actuator and sensing subsystems. An integrated design and control methodology proposed in Part I of this paper proposed varying design topology and controller order for meeting performance requirements of the closed-loop controlled system. A detailed set of steps was given for meeting requirements such as a desired static or dynamic load-capacity, bandwidth, or range of motion. In this part of the paper, an application case study for a practical precision positioning and alignment system is worked out to illustrate the steps involved in using the proposed methodology. The details of optimization problem formulation and solutions for design and control are presented. The outcome of the exercise is a novel design topology, with it shape and size optimized, and a controller synthesized such that a desired control bandwidth and design requirements of strength and modal separation are met.
\end{abstract}

Keywords: Flexure-based mechanisms, Nanopositioning, Topology Generation, Synthesis.

\section{INTRODUCTION}

Precision positioning applications built around conventional bearings (such as sliding contact or rolling contact bearings) are often hindered by friction, backlash, hysteresis, and other motion non-linearities. Flexure-based mechanisms rely inherently on the beam-like material behavior, and can be designed to show linear elastic behavior free of such motion non-linearities, allowing for nanoscale resolutions over small ranges of motion on the order of a few millimeters.

In Part I of this paper, a novel methodology integrating design and control considerations was presented. The key distinction of this "co-design" approach is that the design is iterated over topologies and not just parameters within a selected topology. The topology generation is aimed as a valuable addition to the design toolkit, facilitating novel designs that could not have been conceived otherwise. The parameters within any particular topology could be adjusted at a subsequent phase through a detailed shape and size optimization. Further, a novel controller parameterization is used to vary the controller order while directly tuning the sensitivity function to a desired form.

In this Part II of the paper, we detail an application case study of a precision positioning and alignment system

\footnotetext{
$\overline{1 \text { Corresponding author: svijay@mit.edu }}$
}

containing a flexure module driven by a precision actuator such as a piezoelectric actuator, so that a desired set of performance requirements are met. The rest of the paper is organized as follows. An overview of the problem is presented in Section 2. Application of detailed steps of the integrated design and control methodology is described in Section 3. Simple lumped parameter models used for deriving parametric relations between performance requirements and parameters of topologies are discussed in Section 4. An optimization problem formulated for the system is presented in Section 5 and the results are discussed in Section 6, highlighting the demonstrated advantages of varying the topology and the controller order in the proposed "co-design" approach. The paper concludes with a summary of contributions and directions for future research in Section 7.

\section{PROBLEM OVERVIEW}

In this section, we work out in simulation an example application to illustrate the integrated design and control methodology presented in Part I of this paper and highlighted briefly above. A simple, yet practical, positioning system example with a flexure-based mechanism driven by a piezoelectric actuator is considered. A broad overview of the rest of the section is as follows. We present a generic problem description, and then specify a set of critical 
performance requirements for the problem. The methodology is applied to first generate a set of design concept topologies. Based on design screen tests, a few topologies are ruled out. An optimization problem is formulated in terms of a desired cost function and a set of physical constraints. Design topologies passing the screening test are then input to the optimization problem. A MATLABbased optimizer is used to fine-tune the shape and size of topology candidates. If the design or control cost function cannot be physically obtained, an optimal solution cannot be obtained and the topology is discarded.

\subsection{Description}

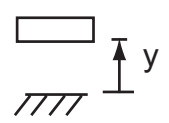

Fig. 1. Schematic diagram showing a positioning system example. The goal is to vary the gap $z$ over a large range of motion and control bandwidth.

Consider the precision positioning problem shown schematically in Fig. 1. The objective is controlling a relative separation $z$ between a moving surface and a fixed surface to form a controllable gap. Such a gap can be used to study physical phenomena at sub-micron scales, such as radiative heat transfer or force interactions such as Casimir forces that occur between metals [2]. Other example applications include size-based filtration for macromolecular separations [1] and characterization of electrochemical properties of gas and liquid molecules [3].

A piezoelectric stack actuator with a lever amplification mechanism is suggested for generating a large displacement range on the order of $100 \mu \mathrm{m}$ required for the gap $z$. A schematic diagram showing the concept of a lever mechanism with piezoelectric actuator is presented in Fig. 2. The piezoelectric stack, shown as generating an input displacement $y_{i n}$, pushes a lever at a distance $L_{a}$ away from its pivot. The gap is formed at the distal end, a distance $L_{s}$ away from the pivot, where the lever output displacement $y_{\text {out }}$ is sensed with a laser interferometer. For small-angle motions of the lever about the pivot, the output displacement $y_{\text {out }}$ for a input displacement $y_{\text {in }}$ is given as

$$
y_{\text {out }}=y_{\text {in }} \frac{L_{s}}{L_{a}}
$$

Our goal here is to illustrate the design and control methodology for the positioning system conceptualized in Fig. 2, using flexure-based mechanisms in the design to for the pivot. Unlike friction-based bearings, flexure-based bearings are ideal candidates for the pivot owing to their smooth elastic motion and minimal nonlinearities such as backlash or hysteresis.

Before we proceed any further, we need to make a few assumptions for the relevant parameters. First, we assume the piezoelectric stack actuator has a blocking force of
$F_{\text {max }}=850 \mathrm{~N}$ and free deflection $y_{\text {piezo,max }}$ of $18 \mu \mathrm{m}$. The static force-deflection characteristic of the actuator is shown in Fig. 3. In our design, we use two piezoelectric stack actuators held mechanically in series, so that their displacements add up to cause the net displacement input. For a maximum displacement $y_{\text {in }}$ of $18 \times 2=36 \mu \mathrm{m}$, we need to meet a target of $100 \mu \mathrm{m}$ at the output. Let us assume reasonable values for the distance of the sensor from the pivot $L_{s}=2 \mathrm{in}$, and distance from actuator to pivot $L_{a}=0.5 \mathrm{in}$. This results in an amplification ratio of 4 , and a resultant maximum output displacement $y_{\text {out }}$ of $144 \mu \mathrm{m}$, which satisfies our target displacement of $y_{\text {desired }}=100 \mu \mathrm{m}$. Basing on the stiffness of the structure, the applied force may vary, and the net displacement input can be smaller, so the extra buffer of $44 \mu \mathrm{m}$ is desirable.

Note that the simple model of Fig. 2 also depicts the simplified dynamics of a disk drive actuator subsystem example given in [4]. As we will be discussing towards the end of this chapter, in the example of [4], the geometry of a design is altered to improve control performance. Therein, changing the geometry involves changing the parameters within a selected topology. Here, as an alternative approach, we explore the option of varying the design topology to improve on the control performance. The case in which parameters within a design topology are varied is covered in our broad methodology. ${ }^{2}$

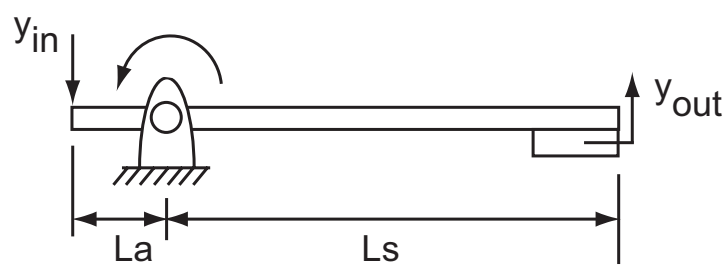

Fig. 2. Schematic diagram of a piezoelectric actuator with lever amplification mechanism.

\subsection{Problem Statement}

The problem statement for applying the proposed integrated design and control methodology to the example of the positioning system of Fig. 2 is as follows:

Given a lever amplification mechanism of Fig. 2 with the following parameters:

(ii) output displacement $y_{\text {out }}$ measured at a distance $L_{s}=$

2 As explained in Section 4, where we discuss the dynamics of a few designs represented by the simple model of Fig. 2, we motivate the need for altering the design topologies so as to move the nonminimum phase zero outside the range of frequencies of interest. Our approach of integrated design and control is implemented for achieving this feature. In the example of disk drive actuator system given in [4], altering geometry of the given topology eliminates nonminimum phase zeros. In a actual multi-DOF system, given many constraints on geometry, and design requirements, both (i) varying parameters within a topology and (ii) varying the topology (and parameters within each topology) should be explored. As we have seen earlier, the integrated design and control methodology applied in Section 3 covers both these cases. 


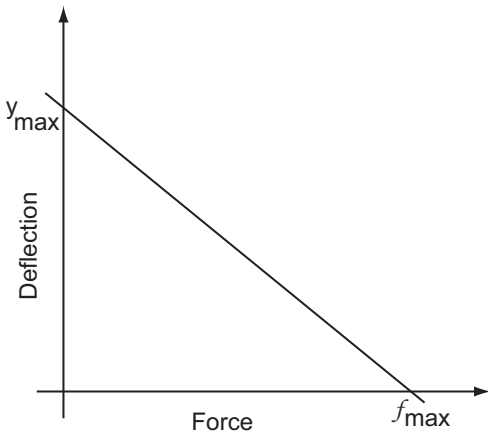

Fig. 3. Typical static force-deflection characteristic curve of a piezoelectric stack actuator. The piezoelectric stack actuator we select in this application has a maximum force capacity (blocking force) $F_{\max }=$ $850 \mathrm{~N}$ and free deflection $y_{\max }$ of $18 \mu \mathrm{m}$.

2 in from the pivot.

(iii) input displacement $y_{\text {in }}$ provided at a distance $L_{a}=$ 0.25 in from the pivot.

(iv) a piezoelectric stack actuator with a blocking force $F_{\max }=850 \mathrm{~N}$ and free deflection $y_{\text {piezo,max }}$ of $18 \mu \mathrm{m}$.

\section{Design a flexure-based pivot that meets the per-} formance requirements given in Table 1.

\section{IMPLEMENTATION OF METHODOLOGY}

Given the above parameters for the lever and the piezoelectric stack actuator, we examine the topology, shape-size optimization and control performance of the system when a flexure-based mechanism is used as a pivot for the lever. We now follow the steps of the methodology as presented in Section 3 for the integrated design and control of the flexure-based pivot. For simplicity, we restrict our interest to planar implementations, which can be manufactured relatively easily on an abrasive waterjet or a wire-EDM.

Step 1: Performance specifications: The specifications for the positioning are as given in Table 1 .

Step 2: Design Topology Library Generation: A set of topology concepts derived for flexural pivots are shown in Fig. 5. The idea is to use one of these pivots in the amplification mechanism shown in Fig. 2. One design topology using the flexure-based mechanism in Fig. 5 (c) as a pivot is shown in Fig. 4 .

Table 1. Specifications for 1DOF flexure-based positioning system example.

\begin{tabular}{|l|l|}
\hline Desired Parameter & Value \\
\hline \hline Range of motion, $\max \left(y_{\text {out }}\right)$ & $>50 \mu \mathrm{m}$ \\
\hline Control bandwidth & $>1 \mathrm{kHz}$ \\
\hline $\begin{array}{l}x_{\text {parasitic, } \max \text { Parasitic horizontal }} \\
\text { displacement for } 5^{\circ} \text { uncertainty in } \\
\text { actuator vertical alignment }\end{array}$ & $<1 \mu \mathrm{m}$ \\
\hline Fatigue Performance & $\begin{array}{l}\text { Infinite life, i.e. } \\
\end{array}$ \\
& $\approx 10^{7}-10^{8}$ cycles or more \\
\hline
\end{tabular}

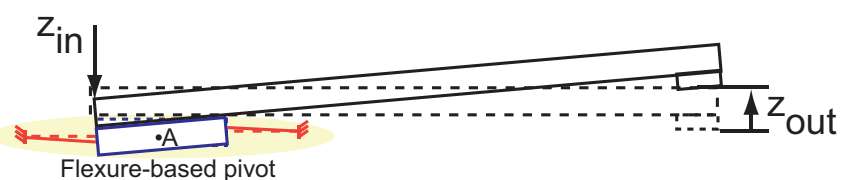

Fig. 4. Design topology showing a flexure-based mechanism as a pivot for the lever amplification mechanism. Since the piezoelectric stack actuator applies a force (and resultant displacement $z_{i n}$ ) offset from the center of mass, the lever rotates about the instantaneous center of rotation $\mathrm{A}$, which forms a virtual hinge or pivoting point in the system. In this figure, flexurebased mechanism of Fig. 5(c) is selected as the pivot. Other design topologies under consideration in our example are those that use the flexure-based mechanisms of Fig. 5 (d)-(j).

The candidate topologies of Fig. 5 were generated as follows. The concepts shown in Fig. 5(a) and (b) are simple examples of a rotational joint achieved with a lumped rotational compliance. While the notch flexure joint in Fig. 5(a) has a localized compliance around its neck, the beam flexure of Fig. 5(b) has a compliance distributed over its length.

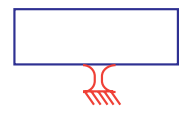

(a)

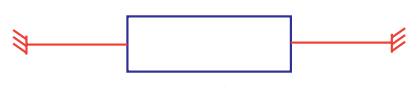

(c)

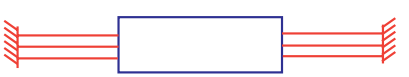

(e)

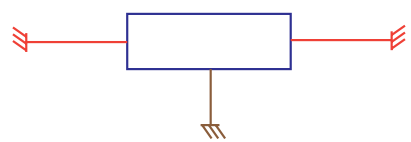

(g)

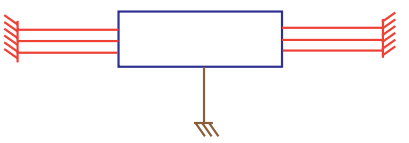

(i)

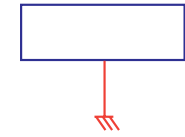

(b)

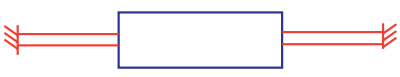

(d)

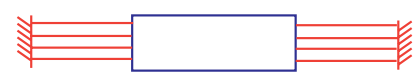

(f)

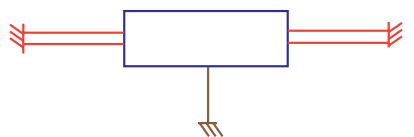

(h)

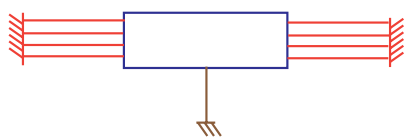

(j)
Fig. 5. A library of candidate design topologies for a flexural pivot.

The rest of the design topologies shown in Figs. 5(c)(j) are obtained as follows. First, we start with a beam flexure as a primitive used to suspend a mass. This primitive is shown in Fig. 6(a). To improve on the 
load-capacity and fatigue performance ${ }^{3}$ of the primitive, we add a beam flexure on the other side of the mass. This enhanced primitive is shown in Fig. 6(b). A symmetric arrangement of the primitives on either side of the mass results in a redundant constraint, allowing for larger load-capacity, while at the same time ensuring under a vertical load the mass moves vertically, without a lateral motion error, as indicated in Fig. 6(b).

Note, however, that there is a second-order effect of over-constraint (the beams fighting with each other), which can be minimized with suitable geometry (for example, longer beam length). An improvement which eliminates the over-constraint is a parallelogram flexure in a folded back configuration [8]. For simplicity, this alternate primitive is not considered in this example.

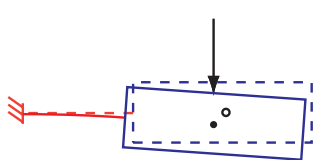

(a)

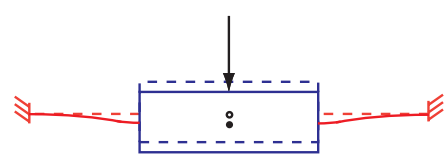

(b)
Fig. 6. Primitive beam flexure shown in (a) is enhanced in its load-capacity by adding a redundant constraint in (b) to produce a double-sided beam flexure primitive.

Further, note also that the primitive flexure choice is not unique to a problem. We converged at the design topologies presented in Fig. 5 (c)-(j) startingS with a beam flexure of Fig. 6(a) as the primitive flexure.

An alternate primitive flexure that can also be considered is a notch flexure. An example double sided notched flexure equivalent of Fig. 6 (b) is shown in Fig. 7. Unlike the beam flexure which has continuous distribution of compliance, the notch flexure has a localized compliance. Designs featuring localized compliances become over-constrained in the presence of manufacturing errors, while those featuring distributed compliances are known to be more robust in the presence of such errors [5]. In this example, hence, we restrict our attention to the case of distributed compliance, i.e. to design topologies derived from beam flexure primitives as against notch flexure primitives.

The concept topology shown in Fig. 5(c) is the double-sided beam flexure primitive of Fig. 6(b). With a force applied at an offset from the center of the mass, a rotational motion can be imparted. The pivoting action achieved with this topology is schematically shown in Fig. 4.

The concept of topology shown in Fig. 5(d) has the same mass now suspended on a parallel stacking of two sets of beam flexures on either side.

3 Both of these are high strength requirements. A large range of motion can be obtained for the same load-capacity with redundant replication of the flexure constraint, instead of reinforcing a single constraint.

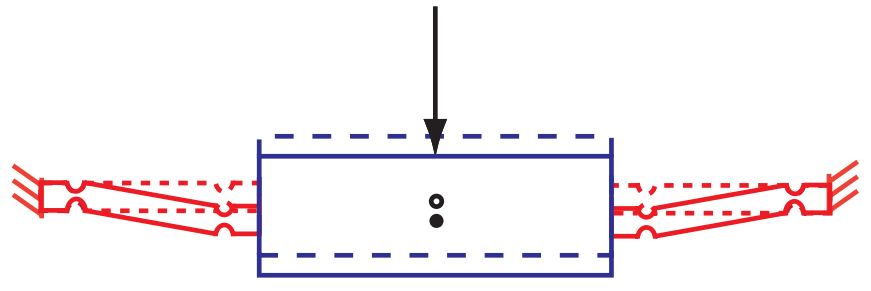

Fig. 7. Double-sided notched flexure primitive. This primitive is the notched equivalent of the double-sided beam flexure of Fig. 6 (b). Unlike the beam flexure, which has a distributed compliance, the notch flexure has a localized compliance at the thin necks of the notch.

On similar lines, the concept of topology shown in Fig. 5(e) and (f) have the same mass now suspended on a parallel stacking of three and four sets, respectively, on either side of the mass.

The concept topology shown in Fig. $5(\mathrm{~g})$ is similar to that of Fig. 5c, but with a rod flexure pinning down the mass at its center of rotation, and hence curbing the trampoline-like $z$-mode. Since the rod flexure has a large rotational compliance compared to axial compliance, this topology corresponds to a large modal separation $S_{Z, \theta}$ between the rotational and vertical DOFs.

The concept topologies shown in Fig. 5(h)-(j) are the similar center-pinned counterparts of Fig. 5(d)(f), respectively.

tep 3: Design Topology Screening: Screening criterion for topologies can be decided according to the needs of the particular application under consideration. There is no unique way to select a screening criterion. Of many possible screening criteria to select the ideal topologies from the candidate topologies of Fig. 5, we select the following criterion that targets the specification of minimal lateral motion errors:

$$
S_{X, Z}>>1
$$

where $S_{X, Z}$ specifies the modal separation of the fundamental $Z$ vibration mode from the $X$ (lateral) vibration mode. We define the modal separation index between any two modes as a ratio of natural frequencies of the modes; a judicious choice of flexure constraints is implemented to maximize the modal separation to minimize parasitic motion errors. The design topologies in Fig. 5(a) and Fig. 5(b) are more compliant in lateral direction than the vertical direction and hence do not satisfy this criterion.

In other words, for a slight vertical misalignment of the piezoelectric stack actuator, there would be a horizontal force component that will likely cause a large lateral error owing to the small lateral stiffness of the design topologies in Fig. 5(a) and Fig. 5(b). Hence, these two design topologies are eliminated.

In contrast, the high lateral stiffness of the beam flexures in Fig. 5(c)-(j) result in a high modal separation with the lateral DOF occurring at much higher frequencies than the vertical DOF. Hence, these ten 
candidates are passed to the subsequent shape and size optimization.

Step 4: Controller Selection/Screening: Of all possible stabilizing controllers, we screen for those that ensure both (i) good command following over frequencies up to $1 \mathrm{kHz}$ and (ii) steady state error of zero for a step input. As will be shown later in Section 4, the plants corresponding to the five nominal design topologies, or plants, have no free integrators. Hence, it is imperative for the controller to have a free integrator in order to satisfy the screening criteria. Many nominal controllers can be constructed to satisfy this screening criterion, such as an integral controller, a proportional-integral controller, a lag controller, and other higher order controllers that have at least one free integrator. In this example, for simplicity, we select a simple integral controller as the nominal controller as given below:

$$
C_{0}(s)=\frac{k}{s}
$$

where $k$ is a nominal gain selected for the given plant to ensure stability of the nominal closed-loop system.

Step 5: Optimization: We follow a sequential approach with the design optimized first, and the optimized design passed to the controller optimization routine. The formulation of the optimization and discussion of optimization results are presented in Sections 5-6. Before we proceed any further, we need to derive lumped parameter models and extract parametric relations needed for the optimization problem formulation.

\section{LUMPED PARAMETER MODELING}

A lumped parameter model for the design topologies using flexure-based pivots of Figs. 5(c)-(f) is shown in Fig. 8. In this model, the flexure-based pivot

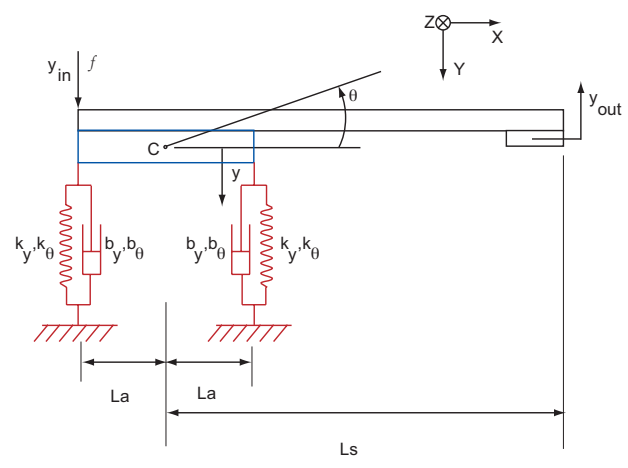

Fig. 8. Lumped parameter model for depicting dynamic behavior of topology concepts using flexure-based mechanisms of Fig. 5(c)-(f) as pivots in the 1-DOF positioning system.

is shown as a lumped mass suspended on its either side by flexures having a lumped linear stiffness $k_{y}$ and a rotational stiffness $k_{\theta}$. The pivoting point, or the instantaneous center of rotation of the system is the center of the mass, denoted by $C$ in the figure. For a downward deflection $y$ of the center $C$ and an angle of rotation $\theta$ of the mass and the lever (about an axis $\mathrm{Z}$ perpendicular to the page and passing through $C$ ), the equations of motion for the system are:

$$
\begin{aligned}
m \ddot{y} & =f-2 b_{y} \dot{y}-2 k_{y} y \\
J \ddot{\theta} & =\tau-2 b_{\theta} \dot{\theta}-2 k_{\theta} \theta \\
f & =f_{\max }-\frac{f_{\max }}{y_{\max }} y_{i n}
\end{aligned}
$$

where $m, J$ are the total mass and moment of inertia about $Z$ axis passing through $C, \tau=f L_{a}$ is the moment applied by the force $f$ applied by the piezoelectric stack actuator. Assuming lightly damped harmonics, damping factors $b_{y}$ and $b_{\theta}$ denoting small damping in the flexures are used.

From the kinematics, as shown in Fig. 9, the output displacement $y_{\text {out }}$ in terms of the downward deflection $y$ of the instantaneous center of rotation and the angle of rotation $\theta$ is given as below:

$$
y_{\text {out }}=-y+L_{s} \theta
$$

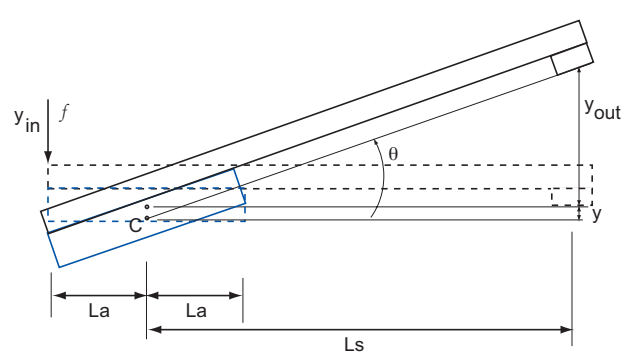

Fig. 9. Schematic diagram showing kinematic relation between the output displacement $y_{\text {out }}$, the downward displacement $y$ of at the center of rotation $C$ and an angle of rotation $\theta$. For small angle motions, since $y_{\text {out }}+y=L_{s} \theta$, we have $y_{\text {out }}=L_{s} \theta-y$.

The transfer function $\frac{Y_{\text {out }}(s)}{F(s)}$ between the applied force input $F(s)$ from the piezoelectric stack actuator to the output displacement $Y_{\text {out }}(s)$ is given by:

$$
\begin{aligned}
\frac{Y_{\text {out }}(s)}{F(s)} & =-\left\{\frac{1}{m s^{2}+2 b_{y} s+2 k_{y}}\right\} \\
& +L_{s}\left\{\frac{L_{a}}{J s^{2}+2 b_{\theta} s+2 k_{\theta}}\right\}
\end{aligned}
$$

The first term in Eq. (8) corresponds to the contribution of the fundamental vertical $(y)$ mode of the flexural pivot as seen at the output displacement measurement. Similarly, the second term corresponds to the contribution of the fundamental rotational $(\theta)$ mode of the flexural pivot. Note the negative sign premultiplying the vertical mode. This means that, at the output, the difference of these two modes is being measured.

A lumped parameter model for the design topologies using flexure-based pivots of Figs. 5(g)-(j) is 
shown in Fig. 10. This model is the same as the model of Fig. 8 except for the enhanced lumped stiffness components at the center $A$. The rod flexure adds a high vertical stiffness $k_{0 y}$, and a mild rotational stiffness $k_{0 \theta}$. The equations of motion for the enhanced system are:

$$
\begin{aligned}
m \ddot{y} & =f-b_{y}^{\prime} \dot{y}-k_{y}^{\prime} y \\
J \ddot{\theta} & =\tau-b_{\theta}^{\prime} \dot{\theta}-k_{\theta}^{\prime} \theta \\
k_{y}^{\prime} & =k_{y}+k_{0 y} \\
k_{\theta}^{\prime} & =k_{\theta}+k_{0 \theta} \\
y_{\text {out }} & =-y+L_{s} \theta \\
f & =f_{\text {max }}-\frac{f_{\max }}{y_{\max }} y_{\text {in }}
\end{aligned}
$$

After applying Laplace transforms to the equations of motion, the transfer function $\frac{Y_{\text {out }}(s)}{F(s)}$ between the applied force input $F(s)$ from the piezoelectric stack actuator to the output displacement $Y_{\text {out }}(s)$ is given by:

$$
\begin{aligned}
\frac{Y_{\text {out }}(s)}{F(s)} & =-\left\{\frac{1}{m s^{2}+b_{z}^{\prime} s+k_{y}^{\prime}}\right\} \\
& +L_{s}\left\{\frac{L_{a}}{J s^{2}+b_{\theta}^{\prime} s+k_{\theta}^{\prime}}\right\}
\end{aligned}
$$

Note again the negative sign pre-multiplying the vertical mode. This means that at the output, as in the case of the lumped parameter model of Fig. 8, the difference of these two modes is being measured.

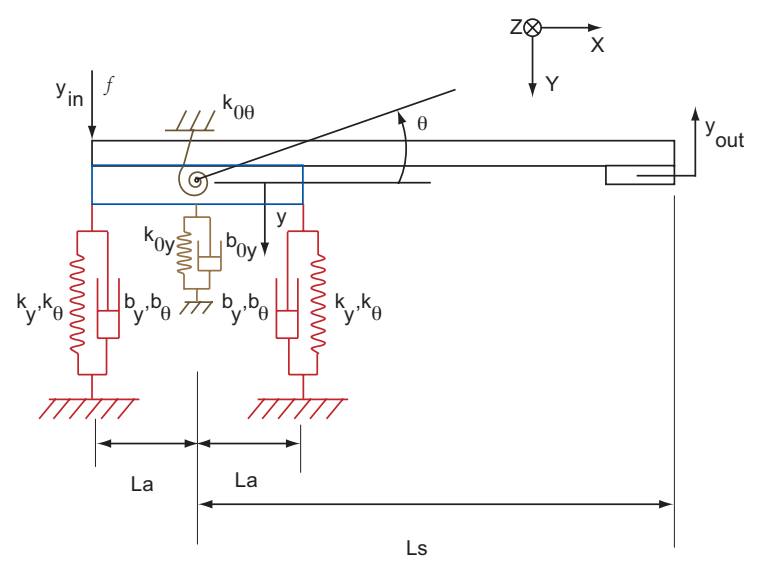

Fig. 10. Lumped parameter model for depicting dynamic behavior of topology concepts using flexure-based mechanisms of Fig. $5(\mathrm{~g})-(\mathrm{j})$ as pivots in the $1-\mathrm{DOF}$ positioning system.

\section{OPTIMIZATION: PROBLEM FORMULATION}

\section{Optimization parameters}

For the flexure-based pivots of Figs. 5(c)-(f), the optimization parameters are selected as the length $\ell$ and thickness $h$ of the beam flexures. For the case of Fig. 5(g)-(j), another variable, the length $\ell_{0}$ of the rod flexure is also considered.

\section{Constraints}

The geometry/dimensional bounds on the parameter for a given footprint of the flexure-based pivot include

$$
\begin{gathered}
0.25 \text { in } \leq \ell \leq D_{\max }-2 L_{a}=D_{\max }-1 \text { in } \\
0.05 \text { in } \leq h \leq 0.3 \text { in } \\
0.25 \text { in } \leq \ell_{0} \leq 2 \text { in }
\end{gathered}
$$

Let $\sigma$ be the maximum stress in the beam flexures, $\sigma_{r}$ the maximum stress in the rod flexure, $y_{i n}$ the displacement input from the piezoelectric stack actuator, $y_{\text {out }}$ is the output displacement, and $x_{\text {out }}$ the lateral motion error. The constraints used in the optimization are:

$$
\begin{aligned}
\text { Constraint }: & \sigma<\sigma_{\max } \\
& \sigma_{r}<\sigma_{\max } \\
& y_{\text {desired }}<y_{\text {out }} \\
& y_{\text {in }}<y_{\text {piezo, } \max } \\
& x_{\text {out }}<x_{\text {parasitic }, \text { max }}
\end{aligned}
$$

where $\sigma_{\max }$ is the maximum allowed stress in the material, $y_{\text {desired }}$ is the $100 \mu \mathrm{m}$ output displacement requirement, $y_{\text {piezo, } \max }$ is the maximum piezoelectric stack deflection, and $x_{\text {parasitic,max }}$ is the maximum allowed parasitic lateral displacement. In order that the mechanism can withstand an infinite number of stress cycles, the maximum stress The cost function is defined for maximizing the output displacement and minimizing the lateral error motion as follows:

$$
\text { Cost : } 0.5 \frac{y_{\text {out }}}{y_{\text {min }}}-0.5 \frac{x_{\text {min }}}{x_{\text {out }}}
$$

Using parametric relations derived useing the models of Section 4, a constrained minimization problem was set up in MATLAB using fmincon with an optimization parameter vector $\mathbf{U}=\left[\ell ; h ; \ell_{0}\right]$ with an initial guess $\left.U_{0}=\ell^{0} ; h^{0} ; \ell_{0}^{0}\right]$, and the bounds $U_{\min }=[0.25 \mathrm{in} ; 0.05 \mathrm{in} ; 1 \mathrm{in}]$ and $U_{\max }=\left[D_{\max }-\right.$ $\left.2 L_{a} ; \frac{D_{\max }-2 L_{a}}{10} ; 2 \mathrm{in}\right]$,

the cost function given in Eq. (21), the constraint conditions given in Eq. (20) using the constrained minimization solvers of MATLAB.

The details of the controller optimization are as follows:

\section{Control Parameter}

A control parameter to tune in our optimization is the parameter transfer function $Q(s)$.

\section{Cost function}

For good command following we choose a weight $W_{s}(s)$, such that a norm, say the $\infty$-norm, of the weighted sensitivity transfer function $W_{s}(s) S(s)$ is optimized as follows:

$$
\left\|W_{s}(s) S(s)\right\|_{\infty} \leq 1
$$

The weight $W_{s}(s)$ is chosen as [7]

$$
W_{s}(s)=\frac{\left(s+M \omega_{d}\right)\left(s+f M \omega_{d}\right)}{s\left(s+f M^{2} \omega_{d}\right)}
$$


Table 2. Results of Optimization of Design and Control for the case of topologies of Fig. 5(g)(j) used as flexure-based pivot.

\begin{tabular}{|c|c|c|c|}
\hline Topology & $\begin{array}{l}\text { Parameter } \\
\text { Values }\end{array}$ & $\begin{array}{l}\text { Range } \\
(\mu m)\end{array}$ & $\begin{array}{l}\text { Control } \\
\text { Band- } \\
\text { width }(\mathrm{Hz})\end{array}$ \\
\hline Fig. 5(g) & $\begin{array}{l}\ell=1.5 \mathrm{in} \\
h=1.25 \mathrm{in} \\
\ell_{0}=1.00 \mathrm{in}\end{array}$ & 124.5 & 1194 \\
\hline Fig. 5(h) & $\begin{array}{l}\ell=1.5 \mathrm{in} \\
h=0.05 \mathrm{in} \\
\ell_{0}=1.00 \mathrm{in}\end{array}$ & 141.1 & 1194 \\
\hline Fig. 5(i) & $\begin{array}{l}\ell=1.5 \mathrm{in} \\
h=0.05 \mathrm{in} \\
\ell_{0}=1.00 \mathrm{in}\end{array}$ & 139.7 & 1194 \\
\hline Fig. 5(j) & $\begin{array}{l}\ell=1.5 \mathrm{in} \\
h=0.05 \mathrm{in} \\
\ell_{0}=1.00 \mathrm{in}\end{array}$ & 138.4 & 1194 \\
\hline
\end{tabular}

with $M=1.5, f=10$, and $\omega_{d}=1.5 \mathrm{kHz}$ is the desired closed-loop system bandwidth. To make the controller $C(s)$ obtained with this choice of $Q(s)$ strictly proper, a filter with two first-order poles at $200 \times \omega_{d}$ is used to ensure a roll-off at high frequencies.

\section{RESULTS AND DISCUSSION}

The results of the optimization are presented in Table 2 . An optimal solution was found for the case of flexurebased pivots of Fig. 5(g)-(j) for both the design and control optimization problems. A grid of 1000 points uniformly spread in the three-dimensional optimization parameter space $\mathbf{U}_{\min } \leq \mathbf{U} \leq \mathbf{U}_{\min }$ were each selected as an initial guess for the optimization. The values of the optimization parameters that gave the best optimal solution, i.e. lowest cost function value with no violation of constraints within a numerical tolerance

The optimization problem resulted in an infeasible solution in both design and control problems for the case of flexurebased pivots of Fig. 5(c)-(f). Note that the infeasible solution was an outcome of optimization at each of the 1000 grid points chosen in the three-dimensional optimization parameter space $\mathbf{U}_{\min } \leq \mathbf{U} \leq \mathbf{U}_{\text {min }}$, for a maximum number 200 sequential quadratic programming iterations at every grid point.

The design topologies of Fig. 5(c)-(f) turn out to be infeasible, the reason for which is discussed as follows. First, it can be shown that these topologies are hindered in their control performance by the presence of non-minimum phase zeros.

Fig. 11 shows a typical pole-zero plot for the open-loop plant. Size or shape-optimization accomplished by varying flexure length $\ell$ or thickness $h$ cannot move the open-loop zeros out of the right half plane.

The presence of the right half-plane zero limits the bandwidth of these design topologies to about half the frequency of the zero, and hence the desired bandwidth of $1 \mathrm{kHz}$ cannot be achieved [7]. The limitation on the

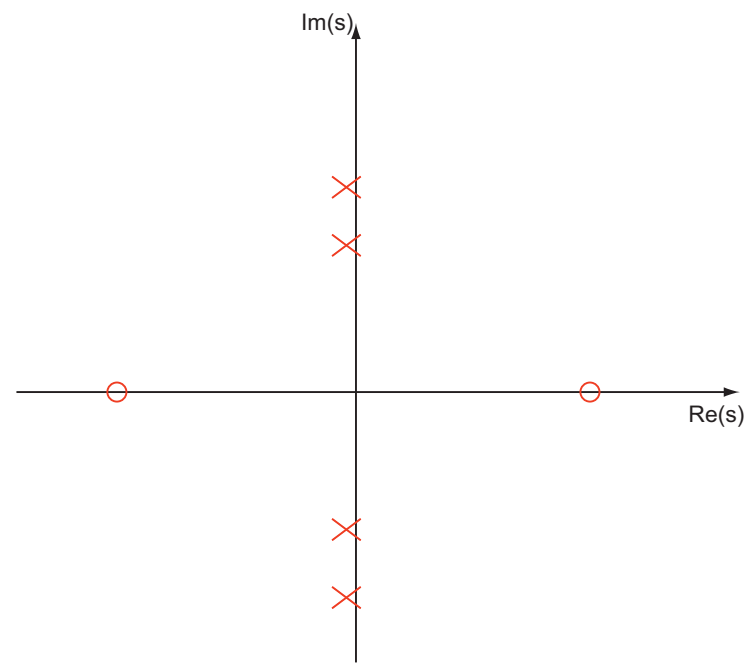

Fig. 11. Pole-zero plot of open-loop plant corresponding to design topologies using flexure-based mechanisms of Fig. 5(c)-(f) as pivots in the 1-DOF positioning system. The zeros of the system are on the real axis and symmetric about the imaginary axis, hence resulting in a non-minimum phase behavior.

bandwidth can be explained from a root-locus viewpoint: higher controller gains resulting from high bandwidth requirements will result in the closed-loop system poles moving toward the right-half-plane zero, and hence result in instability. To ensure stability, the gains have to be limited, and hence the bandwidth has to be limited.

The bound on the bandwidth for the topologies achieved with the flexure-based pivots of Figs. 5(c)-(f) can be derived as follows [7]. Let $\omega_{0}$ denote the right half plane zero of the system. Since the system has no right half plane poles, the sensitivity transfer function $S(s)$ should obey the following constraint:

$$
|| W_{s}\left(\omega_{0}\right) S\left(\omega_{0}\right) \|_{\infty} \geq\left|W_{s}\left(\omega_{0}\right) S\left(\omega_{0}\right)\right|=\left|W_{s}\left(\omega_{0}\right)\right|
$$

where, we have used the fact that the sensitivity transfer function assumes a value of 1 at the frequency of the zero. At this frequency, the condition on the upper bound of the weighted sensitivity given in Eq. (22) reduces to the following:

$$
\left|W_{s}\left(\omega_{0}\right)\right|<1
$$

Using the weighting filter $W_{s}$ given in Eq. (23), the above inequality assumes the form given below:

$$
\left|\frac{\left(\omega_{0}+M \bar{\omega}_{d}\right)\left(\omega_{0}+f M \bar{\omega}_{d}\right)}{\omega_{0}\left(\omega_{0}+f M^{2} \bar{\omega}_{d}\right)}\right|<1
$$

with $M=1.5 f=10$, an upper bound on the achievable control bandwidth $\bar{\omega}_{d}$ is:

$$
\bar{\omega}_{d}<\left(1-\frac{1+f}{M f}\right) \omega_{0}=\frac{4}{15} \omega_{0}
$$


For the flexure-based pivots of Fig. 5(c)-(f) the maximum value for the frequency of the non-minimum phase zero was found to be $\max \left\{\omega_{0}\right\}=944.49 \mathrm{~Hz}$ over the optimization parameter space, which then results in a value for the maximum achievable bandwidth from Eq. (27) to be $251.73 \mathrm{~Hz}$. This value is much lower than our target bandwidth of $1000 \mathrm{~Hz}$ and hence these design topologies cannot meet the control performance requirements. Further the design constraint of infinite fatigue life were found in the optimization to be too stringent on the beam flexures. Since the central rod flexure is not available in these topologies all the applied load is taken by the beam flexures, which have a limited stress handling capability owing to the material yield limit. Hence, the flexure-based pivots of Fig. 5(c)-(f) need to be discarded in our integrated design and control methodology.

One strategy to tackle the non-minimum phase zero is to move it far beyond the frequencies of interest.

In the topologies of Figs. 5(g)-(j) the rod flexure stiffening the trampoline-like $y$ mode of the pivot allows for pushing the non-minimum phase zeros on to the imaginary axis. The resulting system is minimum-phase with no bandwidth limitations imposed by their presence.

Of all the designs, the design topology with four beams in Fig. 5(h) has the largest vertical range. The design topology of Fig. 5(j) has the lowest lateral displacement error since it has the largest stiffness in $X$ direction. Surprisingly, the design topology of Fig. $5(\mathrm{~g})$ does not have the largest vertical range. This result is not obvious, and the optimal parameter vector is different from the rest of the topologies. One possible reason is that since there are fewef beam flexures to carry the load, the stress in the material is a limiting factor. This in fact is reflected in the optimal width of $h=1.25 \mathrm{in}$ for this topology, as against $h=0.05$ in for the rest of the topologies.

All the design topologies of Fig. 5(g(-) $\mathrm{j})$ meet the control performance requirement of $1000 \mathrm{~Hz}$ bandwidth. The control performance of the design topology with the the flexure-based pivot of Fig. 5(j) is shown in Fig. 12 in terms of the sensitivity transfer function. The nominal sensitivity transfer function resulting from a nominal controller $C_{0}(s)=\frac{1000}{s}$ has a low bandwidth, while the desired sensitivity has a bandwidth of $1000 \mathrm{~Hz}$, a rollon of $40 \mathrm{db} / \mathrm{dec}$. Under a novel control parameterization approach, with a model-matching procedure, the controller parametric transfer function $Q(s)$ was designed such that the sensitivity transfer function closely achieves the desired sensitivity transfer function of $\frac{1}{W_{s}(s)}$. Hence,

$$
S(s)=\frac{1}{W_{s}(s)}=S_{0}(s) Q(s)
$$

For $S_{0}(s)=\frac{1}{1+P C_{0}(s)}$, where $P(s)=\frac{Y_{\text {out }}(s)}{F(s)}$ and $C(s)=$ $\frac{1000}{s}, \mathrm{Q}(\mathrm{s})$ was found to be:

$$
\begin{aligned}
Q(s) & =\frac{Q_{\text {num }}(s)}{Q_{\text {den }}(s)} \\
Q_{\text {num }}(s) & =s^{4}+2.121 \times 10^{5} s^{3}+1.73 \times 10^{8} s^{2} \\
& +3.665 \times 10^{13} s+3.716 \times 10^{10} \\
Q_{\text {den }}(s) & =s^{4}+1.55 \times 10^{5} s^{3}+2.172 \times 10^{9} s^{2} \\
& +2.668 \times 10^{13} s+3.445 \times 10^{17}
\end{aligned}
$$

To ensure a roll-off behavior for the resulting controller, a 2-pole low pass filter with coincident poles at $s=$ $-200 \omega_{d}$ was multiplied with the controller. The resulting sensitivity transfer function matches well with the desired sensitivity transfer function as shown in Fig. 12.

For comparison, a robust controller designed with a mixedsensitivity criterion (allowing for tuning both sensitivity and complementary sensitivity) was also simulated, using mixsyn routine in MATLAB Robust Control Toolbox. The sensitivity transfer function obtained with this approach shows in Fig. 12 a flat profile at low frequencies and a roll-on of $40 \mathrm{~dB} / \mathrm{dec}$ starting at about $0.1 \mathrm{rad} / \mathrm{s}$.

The peak response of the sensitivity transfer function obtained with our method has about $2 d B$ taller peak than obtained from the mixed-sensitivity method, which implies a relatively poorer robustness to uncertainties in the positioning system. The closed-loop system sensitivity developed with our method shows a bandwidth of about $1194 \mathrm{~Hz}$, while that of the mixed-sensitivity approach shows $1430 \mathrm{~Hz}$. The mixed-sensitivity approach resulted in a controller of $8^{\text {th }}$ order, and has a lower peak in the sensitivity transfer function, indicating better robustness, which can be owed to the higher order of this controller.

With performance comparable to a well-established routine like the mixed-synthesis controller of MATLAB Robust Control Toolbox, the controller parameterization introduced here allows for tuning directly the sensitivity transfer function, which plays an important role in addressing lightly damped harmonics of flexible structures. The details of the controller parameterization are not covered here and will be part of a future paper from our group.

In a nutshell, we have converged at a final design topology that meets the specified performance requirements of a bandwidth of greater than $1000 \mathrm{~Hz}$ and a range of motion exceeding $50 \mu \mathrm{m}$, with infinite stress-cycle life. Further, we have a systematic procedure to develop the topologies, screen them for desired features, and optimize them while dealing with dimensional and material constraints. An outcome of this exercise is the apriori identification of non-minimum phase zeros in flexure-based mechanism designs. Non-minimum phase zeros occur whenever noncollocated actuator and sensor arrangements are implemented. Avoiding the non-minimum phase zero may require reconsidering where to measure relative to where we actuate the system. In our case, we chose not to vary the actuator or sensor location [6], or change the geometry 


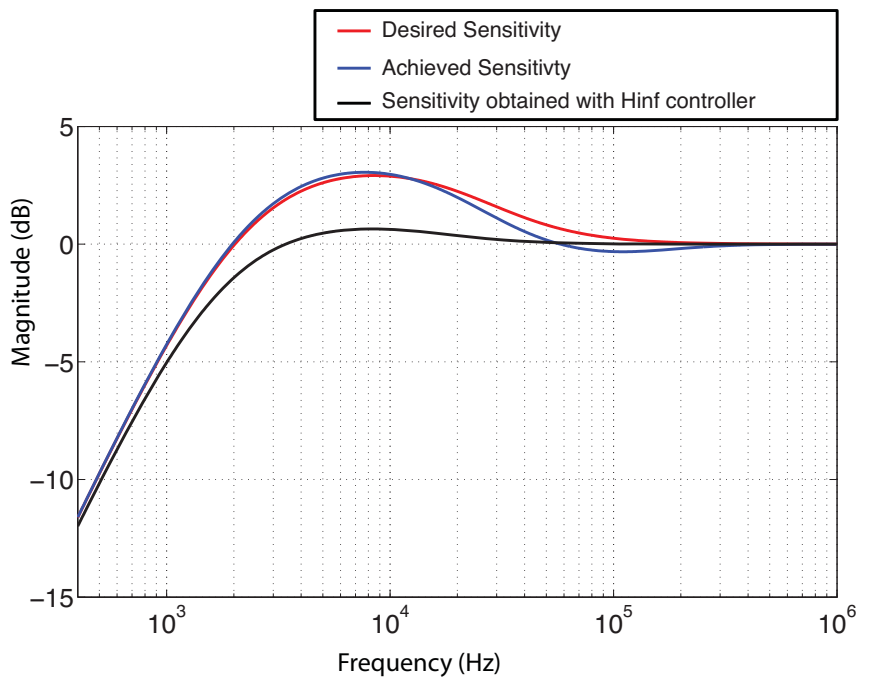

Fig. 12. Comparison of magnitude response of desired and achieved sensitivity transfer function designed with a model-matching matching procedure. A corresponding response obtained for the case of mixed-sensitivity design is also shown.

of the design [4], but rather design the mechanism to be stiffened beyond the bandwidth of interest while still meeting the desired motion requirements. This was possible because of judicious design of topology in terms of flexure constraints, while satisfying strength and dynamic performance requirements.

\section{SUMMARY}

In this paper, we presented a flow chart for iterating on design (plant) and controller to achieve a desired closedloop system specification. It is emphasized that iterating a design is not just about fine-tuning shape and size of a particular design configuration. Instead, we need to iterate over design topologies and controller order. An example of a flexure-based 1-DOF positioning system was worked out to show the integrated design and control methodology. Parametric relations were derived from lumped parameter models to formulate an optimization problem over the design space and the control performance space. The methodology was worked out step-by-step to cover (i) generation of design topologies (ii) screening of topologies for obvious design choices that cannot work for the given application, (iii) optimization formulation in terms of design parameters, cost functions, and equality and inequality constraints, and (iv) controller generation based on model-matching of a sensitivity transfer function. The infeasibility of a set of topologies was explained by the presence of non-minimum phase zeros that limit the achievable control bandwidth. Based on the intuition gained from this exercise, we also suggested a new screening guideline for checking for non-minimum phase zeros possible for a design topology along with an actuator and sensor placement. This example provides a practical 1DOF positioning system application and provides a guided approach to converge at a novel design topology that meets all the requirements.

\section{REFERENCES}

[1] White JR, "The nanogate: nanoscale flow control," Ph.D. dissertation, Cambridge, MA: Massachusetts Institute of Technology, Department of Mechanical Engineering, June 2003.

[2] Shilpiekandula V, "Progress through Mechanics: Small-scale Gaps," Mechanics (Publication of the American Academy of Mechanics), vol. 35, no. 9-10, pp. 3-6, Sep-Oct 2006.

[3] White J, Ma H, Lang J, Slocum AH, "An Instrument to Control Parallel Plate Separation for Nanoscale Flow Control," Review of Scientific Instruments, vol. 74, no. 11, November 2003.

[4] Subrahmanyan PK, "Control and design interactions in precision machines," Tutorial Notes, American Society for Precision Engineering Annual Conference, Portland OR, Oct 2009.

[5] Awtar S and Sevincer E, 2006, "Elastic Averaging in Flexure Mechanisms: A Multi-Parallelogram Flexure Case-study," In Proceedigns of ASME IDETC/CIE 2006, Philadelphia, PA, Paper No. 99752.

[6] Park JH, Asada H, "Design and control of minimumphase flexible arms with torque transmission mechanisms," IEEE International Conference on Robotics and Automation, pp 1790-1795, May 1990.

[7] Skogestad S and Postlethwaite I, "Multivariable feedback control : analysis and design," Hoboken, NJ, John Wiley, 2005.

[8] Jones RV, "Instruments and Experiences: Papers on Measurement and Instrument Design," John Wiley, 1988. 